\title{
Expression of the microRNA-30 family in pulmonary arterial hypertension and the role of microRNA-30d-5p in the regulation of pulmonary arterial smooth muscle cell toxicity and apoptosis
}

\author{
FAN HU ${ }^{1,2}$, HANMIN LIU ${ }^{1,2}$, CHUAN WANG ${ }^{1,2},{\text { HANWEN } \text { LI }^{3} \text { and LINA QIAO }}^{1,2}$ \\ ${ }^{1}$ Department of Pediatrics, West China Second University Hospital of Sichuan University; ${ }^{2}$ Key Laboratory of \\ Birth Defects and Related Diseases of Women and Children, Sichuan University, Ministry of Education; \\ ${ }^{3}$ State Key Laboratory of Oral Diseases, National Clinical Research Center for Oral Diseases, \\ West China Hospital of Stomatology, Sichuan University, Chengdu, Sichuan 610041, P.R. China
}

Received March 21, 2021; Accepted September 23, 2021

DOI: $10.3892 /$ etm.2021.11031

\begin{abstract}
The biological processes of pulmonary artery vascular smooth muscle cells (PA-SMCs) and pulmonary artery endothelial cells in pulmonary arterial hypertension (PAH) are generally abnormal, with increased levels of proliferation and reduced levels of apoptosis. Although microRNAs (miRNAs/miRs) participate in a number of biological processes in a variety of diseases, such as tumors and infections, studies on the association between miRNAs and PAH are limited. In the present study, blood samples were collected from 6 patients with patent ductus arteriosus. The experimental group included 3 patients with severe $\mathrm{PAH}$, while the control group included 3 patients without PAH. Microarray technology was used to detect the presence of any associated miRNAs. Moreover, a rat PAH model was established via left lung resection followed by monocrotaline injection, involving a total of 8 rats in the PAH group and 8 untreated rat in the control group. Reverse transcription-quantitative PCR was performed to verify the expression levels of the miR-30 family in the animal model. miR-30d-5p mimics and anti-miR-30d-5p were transfected into primary cultured PA-SMCs. Levels of cytotoxicity and cell apoptosis were examined, and Notch-3 expression levels were studied using western blotting. The results of the present study demonstrated that miR-30d-5p expression was downregulated in both patient blood and animal models of the PAH group compared with control groups. In primary cultured PA-SMCs, overexpression of miR-30d-5p attenuated the platelet-derived growth factor-induced toxicity of PA-SMCs, while knockdown of miR-30d-5p resulted in the increased toxicity of PA-SMCs compared with control group.
\end{abstract}

Correspondence to: Professor Lina Qiao, Department of Pediatrics, West China Second University Hospital of Sichuan University, 17 Renminnan Road, Wuhou, Chengdu, Sichuan 610041, P.R. China E-mail: qiaolina@scu.edu.cn

Key words: pulmonary arterial hypertension, microRNA, apoptosis
The apoptosis rate of PA-SMCs increased with the overexpression of miR-30d-5p compared with control group. Moreover, the expression levels of Notch-3 in the miR-30d-5p group were significantly reduced compared with the anti-miR-30d-5p and miR-NC groups. In total, 10 circulating miRNAs that may be associated with PAH were discovered in the present study. Moreover, the expression of the miR-30 family was verified in animal models in vivo, and seven miRNAs in this family were discovered that may be associated with PAH. Additionally, miR-30d-5p was downregulated in both patients with PAH and animal models compared with control groups. Thus, the results of the present study demonstrated that the regulatory mechanism underlying PA-SMCs may be via the Notch-3 signaling pathway.

\section{Introduction}

Pulmonary arterial hypertension (PAH) is a progressive disease characterized by increased pulmonary resistance that leads to right heart failure and in some cases, death $(1,2)$. Thus far, numerous causes have been associated with the mechanisms underlying PAH development. For example, endothelin, nitric oxide and prostaglandin are classic regulatory factors of PAH (3). Pulmonary vascular remodeling is the most notable pathological change in $\mathrm{PAH}$, and pulmonary artery vascular smooth muscle cells (PA-SMCs) and pulmonary artery endothelial cells (PA-ECs) are key factors in vascular activity and remodeling (3). However, the pathogenesis of PAH and associated molecular pathways, such as pulmonary vascular remodeling, and PA-SMC and PA-EC proliferation, are yet to be fully elucidated (4-6).

MicroRNAs (miRNAs/miRs) are a class of small non-coding RNAs (7). miRNAs participate in a number of key biological processes, such as differentiation, cell proliferation and apoptosis. miRNAs exert these biological processes by controlling the $3^{\prime}$ untranslated region of mRNA, which degrades and inhibits the translation of target genes, as well as regulates the expression levels of target genes (8).

In PAH, the biological processes of PA-SMCs and PA-ECs are abnormal, characterized by increased proliferation and 
reduced apoptosis (9-11). miRNAs participate in the regulation of proliferation and apoptosis in numerous diseases. For example, in retinoblastoma miR-675 promotes glioma cell proliferation and motility by regulating the RB1 gene (12). miR-34a and miR-181a have been indicated to participate in apoptosis and oxidative stress in human osteoarthritic chondrocytes (13). miR-21 promotes breast cancer proliferation and metastasis (14). We hypothesized that there may be associations between miRNAs and PAH development. The results of previous studies have revealed that a number of miRNAs participate in the regulation of PAH development, such as miR-29b, miR-138, and miR-222 and miR-204 $(15,16)$. However, the differential expression of miRNAs in PAH is yet to be fully elucidated.

The aim of the present study was to verify the relationship between miRNAs and PAH in order to unravel novel potential therapeutic target for PAH. RNA microarray in patients with patent ductus arteriosus with or without $\mathrm{PAH}$, reverse transcription-quantitative PCR in a PAH animal model, flow cytometry, western blotting, miRNA transfection and MTT assay in primary cultured PA-SMCs were used for this purpose. The differential expression levels of miRNAs in patients with $\mathrm{PAH}$ were investigated. Furthermore, the expression level of the miR-30 family was verified in the lung tissue of rats during the development of PAH. The regulatory functions of miR-30d-5p were also investigated in the toxicity of PA-SMCs.

\section{Materials and methods}

Patient data and blood sample collection. A total of 6 patients (West China Second University Hospital of Sichuan University; Chengdu, China) with patent ductus arteriosus were enrolled in the present study between June 2013 and January 2014. All the patients exhibited no other lung diseases or heart diseases. A total of 3 patients diagnosed with severe pulmonary hypertension by echocardiograms and cardiac catheters were assigned to the $\mathrm{PH}$ group [mean pulmonary artery pressure (PAP), $>70 \mathrm{mmHg}$. A further 3 patients without pulmonary hypertension were included in the control group. The clinical characteristics of all patients are summarized in Table I. Blood samples from the 6 patients were collected for RNA extraction and subsequent experiments. All experiments involving human subjects were approved by the Medical Ethics Committee of West China Second University Hospital of Sichuan University (Chengdu, China; approval no. 2015-010). Written informed consent for using the blood samples of patients was obtained from the parents of the patients.

miRNA differential expression spectrum. Total RNA was extracted from blood samples using PureLink ${ }^{\mathrm{TM}}$ RNA extraction kit (cat. no. 12183020; Thermo Fisher Scientific, Inc.) and purified using mirVana ${ }^{\mathrm{TM}}$ PARIS ${ }^{\mathrm{TM}}$ RNA and Native Protein Purification kit (cat. no. AM1556, Ambion; Thermo Fisher Scientific, Inc.) according to the manufacturer's protocol. RNA integration was determined using an Agilent Bioanalyzer 2100 (Agilent Technologies, Inc.). A sample was considered qualified with an RNA integrity number $>7$.

RNA labeling and array hybridization. miRNAs in total RNA were labeled using the miRNA Complete Labeling and
Hybridization kit (cat. no. 5190-0456; Agilent Technologies, Inc.) according to the manufacturer's protocol. Each slide was hybridized with $100 \mathrm{ng}$ Cy3-labeled RNA using the aforementioned miRNA Complete Labeling and Hybridization kit in a hybridization oven (cat. no. G2545A; Agilent Technologies, Inc.) at $55^{\circ} \mathrm{C}$ and low agitation for $20 \mathrm{~h}$, according to the manufacturer's protocol. Slides were subsequently washed using the Gene Expression Wash Buffer kit (cat. no. 5188-5327; Agilent Technologies, Inc.). An Agilent Microarray Scanner (cat. no. G2565BA; Agilent Technologies, Inc.) was used to scan the slides. The original data were normalized using the Quantile algorithm, Gene Spring Software 11.0 (Agilent Technologies, Inc.).

Animal model. All animal experiments animals were approved by the Medical Ethics Committee of West China Second University Hospital of Sichuan University (Chengdu, China; approval no. 2015-010). Sprague-Dawley (SD) rats were purchased from Chengdu Dashuo Biological Technology Co., Ltd., and raised in specific-pathogen-free conditions. The room temperature was $25^{\circ} \mathrm{C}$ with $50 \%$ humidity. The light and dark cycle was $12 \mathrm{~h}$ each. The rats had free access to food and water. Rats were divided into the following two groups: i) PAH group; and ii) control group.

In the PAH group, a total of 10 male SD rats (weight, 300-400 g; age, 9 weeks) underwent left lung resection and subcutaneous injection of monocrotaline (MCT; $60 \mathrm{mg} / \mathrm{kg}$ ) one week after surgery in order to mimic pulmonary hypertension (1). Rats were anesthetized with an intraperitoneal injection of pentobarbital (30-60 mg/kg) for lung resection, and the duration of the operation was 10-15 min. Following the aforementioned procedures, animal health was monitored daily. In total, 1 rat died 1 day following surgery, and 1 rat died 5 days following surgery. Thus, a total of 8 rats were used in subsequent procedures. At 5 weeks after the drug injection, the rats were used for subsequent studies. The results of our previous study demonstrated that severe PAH formed at 5 weeks following MCT injection (1). Furthermore, the control group consisted of 8 healthy male SD rats (weight, 300-400 g; age, 9 weeks).

PAP was measured through the jugular vein using a transvenous catheter, and animals were subsequently sacrificed. Following the aforementioned anesthesia using an intraperitoneal injection of pentobarbital $(30-60 \mathrm{mg} / \mathrm{kg}$ ), rats were sacrificed by exsanguination via the jugular veins and carotid arteries. Animal death was confirmed by an absence of heart rate and lack of breathing. Lung tissues from both groups were isolated for RNA extraction. The hearts were dissected, and the weight of the right ventricle (RV), left ventricle (LV) and ventricular septum (S) were measured. The right heart hypertrophy index (RVHI) was calculated using the equation: $\mathrm{RV} /(\mathrm{LV}+\mathrm{S})$.

Reverse transcription-quantitative (RT-qPCR). The expression levels of miR-30a-5p, miR-30b-5p, miR-30c-5p, miR-30d-5p, miR-30e-5p, miR-30a-3p, miR-30b-3p, miR-30c-1-3p, miR-30c-2-3p, miR-30d-3p and miR-30e-3p in the lung tissues of the PAH group and control group were verified using RT-qPCR. Total RNA was extracted from lung tissues using TRIzol ${ }^{\circledR}$ reagent (Invitrogen; Thermo Fisher Scientific, Inc.), 
Table I. Clinical characteristics of control patients and patients with PAH.

A, Control patients without PAH

\begin{tabular}{|c|c|c|c|c|c|c|c|}
\hline $\begin{array}{l}\text { Age, } \\
\text { months }\end{array}$ & Sex & $\begin{array}{l}\text { Weight, } \\
\text { kg }\end{array}$ & $\begin{array}{c}\text { Body surface } \\
\text { area, } \mathrm{m}^{2}\end{array}$ & $\begin{array}{l}\text { RVSP, } \\
\text { mmHg }\end{array}$ & $\begin{array}{l}\text { mPAP, } \\
\text { mmHg }\end{array}$ & $\begin{array}{l}\text { PVRI, wood } \\
\text { units } \mathrm{m}^{2}\end{array}$ & $\begin{array}{l}\text { PDA diameter, } \\
\mathrm{mm}\end{array}$ \\
\hline 34 & Female & 14.0 & 0.590 & 27 & 18 & N/A & 2 \\
\hline 47 & Female & 11.5 & 0.503 & 25 & 15 & N/A & 2 \\
\hline 44 & Female & 9.0 & 0.415 & 22 & 14 & N/A & 2 \\
\hline
\end{tabular}

B, Patients with PAH

\begin{tabular}{rlccccc}
\hline $\begin{array}{l}\text { Age, } \\
\text { months }\end{array}$ & Sex & $\begin{array}{c}\text { Weight, } \\
\mathrm{kg}\end{array}$ & $\begin{array}{c}\text { Body surface } \\
\text { area, } \mathrm{m}^{2}\end{array}$ & $\begin{array}{c}\text { RVSP, } \\
\mathrm{mmHg}\end{array}$ & $\begin{array}{c}\text { mPAP, } \\
\mathrm{mmHg}\end{array}$ & $\begin{array}{c}\text { PVRI, wood } \\
\text { units } \mathrm{m}^{2}\end{array}$ \\
\hline 34 & Female & 13.0 & 0.555 & 105 & 75 & 10.83 \\
108 & Male & 26.0 & 1.010 & 116 & 87 & 18.74 \\
49 & Male & 12.0 & 0.520 & 112 & 75 & 21.40 \\
\hline
\end{tabular}

RVSP, right ventricular systolic pressure; mPAP, mean pulmonary artery pressure; PVRI, pulmonary vascular resistance index; PDA, patent ductus arteriesus; $\mathrm{PAH}$, pulmonary arterial hypertension; N/A, not available.

according to the manufacturer's protocol. RT-qPCR was performed using the EzOmics ${ }^{\mathrm{TM}}$ One-Step qPCR kit (cat. no. BK2100; Biomics Biotechnologies Co., Ltd.). A total of $1 \mu \mathrm{l}$ RNA was added to $25 \mu \mathrm{l} \mathrm{EzOmics}{ }^{\mathrm{TM}}$ One-Step qPCR kit components (including $2 \mathrm{X}$ master mix, 50X SYBR Green I, $50 \mathrm{mM} \mathrm{MgCl}_{2}$ and $\left.\mathrm{H}_{2} \mathrm{O}\right), 1 \mu \mathrm{l} \mathrm{EzQuick}{ }^{\mathrm{TM}}$ 50X RT/Taq Mix, $2 \mu \mathrm{l}$ RT primer and $30 \mu \mathrm{l}$ diethylpyrocarbonate- $\mathrm{H}_{2} \mathrm{O}$. The primers were included in the EzOmics ${ }^{\mathrm{TM}}$ miRNA qPCR Detection Primer Set (cat.no.BK1010; Biomics Biotechnologies Co., Ltd.). The thermocycling conditions were as follows: Initial denaturation at $95^{\circ} \mathrm{C}$ for $10 \mathrm{~min}$, followed by 40 cycles of $95^{\circ} \mathrm{C}$ for $15 \mathrm{sec}, 55^{\circ} \mathrm{C}$ for $30 \mathrm{sec}$ and $72^{\circ} \mathrm{C}$ for $30 \mathrm{sec}$. Data were analyzed using the $2^{-\Delta \Delta \mathrm{Cq}}$ method (17) for relative quantification to U6. The primer sequences were as follows: miR-30a-5p forward, 5'-AACGAGACGACGACAGAC-3' and reverse, 5'-TGTAAACATCCTCGACTGGAAG-3'; miR-30b-5p forward, 5'-TGTAAACATCCTACACTCAGCT-3' and reverse, 5'-CAGTGCGTGTCGTGGAGT-3'; miR-30c-5p forward, 5'-ACACTCCAGCTGGGTGTAAACATCCTACA CTC-3' and reverse, 5'-CTCAACTGGTGTCGTGGAGTCG GCAATTCAGTTGAGGCTCAGAG-3'; miR-30d-5p forward, 5'-GCCTATAAACATCCCCGAC-3' and reverse, 5'-GTGCGT GTCGTGGAGTCG-3'; miR-30e-5p forward,5'-TGTAAACAT CCTTGACTGGAAGG-3' and reverse, 5'-CCAGTGCGAATA CCTCGGAC-3'; miR-30a-3p forward, 5'-CCCTGCTCTGGC TGGTCAAACGGA-3' and reverse, 5'-TTGCCAGCCCTGCT GTAGCTGGTTGAAG-3'; miR-30b-3p forward, 5'-GCTGCG GTGTAGACATCTAATAC-3' and reverse, 5'-ATCCAGTGCA GGGTCCGACC-3'; miR-30c-1-3p forward, 5'-ACACTCCAG CTGGGCTGGGAGAGGGTTGTTTACTCC-3' and reverse, 5'-CTCAACTGGTGTCGTGGAG TCGGCAATTCAGTTGA GGGAGTAAA-3'; miR-30c-2-3p forward, 5'-CACGCACTGG GAGAAGGC-3' and reverse, 5'-GTCGTATCCAGTGCAG GGTCCGAGGTATTCGCACTGGATACGAC-3'; miR-30d-3p forward, 5'-TGGTTTTTTAGTATTATTGTTAGTTGT-3' and reverse, 5'-ATACATACAATCCCAACTATTCAAA-3'; miR-30e-3p forward, 5'-ACGCTTTCAGTCGGATGTTTA CAGC-3' and reverse, 5'-GTGCGTGTCGTGGAGTCG-3'; U6 forward, 5'-GCTTCGGCAGCACATATACTAAAAT-3' and reverse, 5'-CGCTTCACGAATTTGCGTGTCAT-3'.

Cell culture. Rat PA-SMCs were isolated and cultured as previously described by Yin et al (18). Immediately after the rats were sacrificed, the pulmonary artery was dissected and removed. The following procedure was performed under aseptic conditions. The connective tissue, tunica intima and tunica adventitia of the artery was eliminated. The rest of the tissue was cut into small pieces $\sim 2 \mathrm{~mm}^{2}$ and transferred to a cell culture flask. A total of $1 \mathrm{~h}$ later, when the tissue attached to the cell culture flask, it was cultured in DMEM (Gibco; Thermo Fisher Scientific, Inc.) with $10 \%$ fetal bovine serum (Gibco; Thermo Fisher Scientific, Inc.). The cells were cultured in a $37^{\circ} \mathrm{C}$ incubator with $5 \% \mathrm{CO}_{2}$.

miRNA transfection. The miR-30d-5p mimics (miR-30d-5p; cat. no. miR10000807-1-5), miR-negative control (NC) mimics (miR-NC; cat. no. miR20000807-1-5), miR-30d-5p inhibitor (anti-miR-30d-5p; cat. no. miR1N0000001-1-5) and miR-NC inhibitor (anti-miR-NC; cat. no. miR2N0000001-1-5) were purchased from Guangzhou RiboBio Co.,Ltd. Primary cultured PA-SMCs were transfected at $37^{\circ} \mathrm{C}$ for $24 \mathrm{~h}$ with the aforementioned miRNAs (100 nM each) using Lipofectamine ${ }^{\circledR} 3000$ reagent (Thermo Fisher Scientific, Inc.) according to the manufacturer's protocol. The cells were harvested $24 \mathrm{~h}$ after transfection and used in subsequent experiments.

MTT assay. An MTT assay was performed using rat PA-SMCs to examine cytotoxicity. Cells were cultured in 96-well plates $\left(5 \times 10^{4}\right)$ and treated with vehicle or $20 \mathrm{ng} / \mathrm{ml}$ platelet-derived growth factor (PDGF; Sangon Biotech, Co., Ltd.) at $37^{\circ} \mathrm{C}$ for 

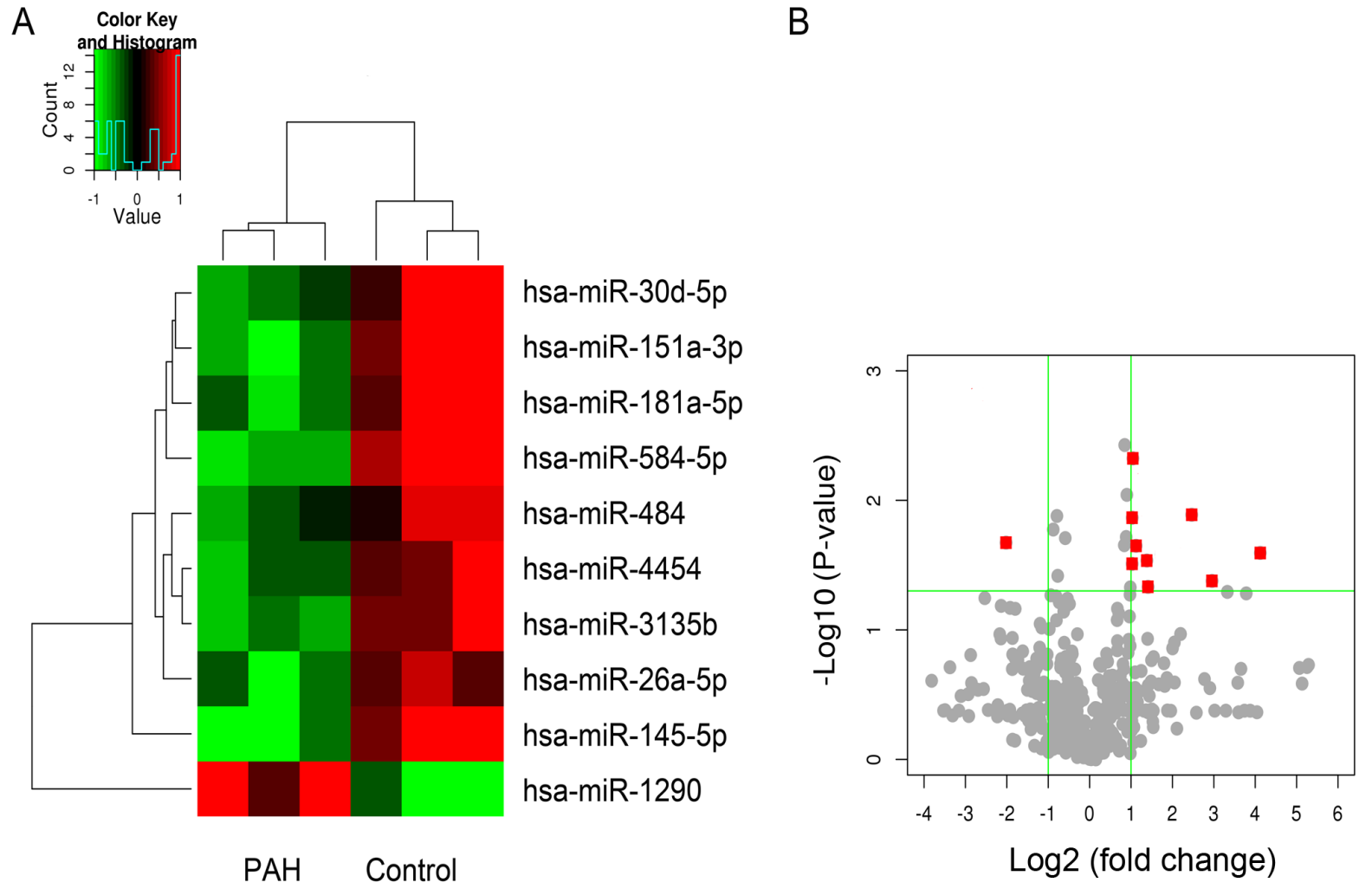

Figure 1. miRNA expression spectrum. (A) Heat map of differential expression of miRNAs in control and PAH groups, using a criteria of $\mathrm{P}<0.05$ and a fold-change $>2$. Green, downregulation; red, upregulation. (B) Volcano plot of miRNAs. Red dots indicate differentially expressed miRNAs matching the criteria of $\mathrm{P}<0.05$ and a fold-change $>2$. miRNA/miR, microRNA; PAH, pulmonary arterial hypertension.

24 h. Cells were further divided into five groups: i) Control, ii) miR-30d-5p, iii) miR-NC, iv) anti-miR-30d-5p; and v) anti-miR-NC. MTT reagent (Sigma-Aldrich; Merck KGaA) was added and cells were incubated for $4 \mathrm{~h}$ at $37^{\circ} \mathrm{C}$. Subsequently, a spectrophotometer was used to assess the formation of colored formazan with dimethyl sulfoxide at $540 \mathrm{~nm}$. All samples were analyzed three times.

Flow cytometry. Flow cytometry was performed using rat PA-SMCs $\left(1 \times 10^{6}\right.$ cells $)$ to examine cell apoptosis. Annexin V-PE and PI (BD Biosciences) were used to stain the PA-SMCs. Apoptotic cells were analyzed using a FACScan flow cytometer (Becton, Dickinson and Company) with Cell Quest software v1.1 (BD Biosciences). The apoptosis rate was calculated as the percentage of early + late apoptotic cells.

Western blot analysis. PA-SMCs were collected $48 \mathrm{~h}$ after transfection. Cells were lysed using phosphatase and proteinase inhibitors. Proteins were extracted with RIPA lysis buffer (Thermo Fisher Scientific, Inc.) and protein concentration was determined via the Bradford method. Proteins (20 $\mu \mathrm{g} / \mathrm{lane}$ ) were loaded in a $4 \% 1.0-\mathrm{mm}$ Bis-Tris gel (Thermo Fisher Scientific, Inc.), and subsequently transferred onto PVDF membranes (Thermo Fisher Scientific, Inc.). No blocking was performed before primary antibody incubation. The membranes were incubated with the primary antibody anti-Notch-3 (1:1,000; cat. no. ab23426; Abcam) at $4^{\circ} \mathrm{C}$ for $1 \mathrm{~h}$. Following primary incubation, membranes were incubated with an anti-rabbit HRP-conjugated secondary antibody (1:2,000; cat. no. ab7090; Abcam) for $1 \mathrm{~h}$ at room temperature.
The integrated optical density of the samples was measured with a visualization reagent (Luminol; Sigma-Aldrich; Merck KGaA) using a Gel-Pro analyzer. An anti- $\alpha$-tubulin antibody (1:1,000; cat. no. ab7291; Abcam) followed by incubation with a secondary antibody (1:2,000; cat. no. ab205719; Abcam) under the same conditions as aforementioned, was used as the loading control.

Statistical analysis. The miRNA microarray analysis results were analyzed using the SBC Analysis System (Shanghai BioChip Co., Ltd.), following the manufacturer's protocol. Additionally, miRNAs with a fold-change $>2$ and $\mathrm{P}<0.05$ were considered to indicate a statistically significant difference. All other data were analyzed using SPSS software version 23.0 (IBM Corp.). Data following normal distribution were presented as the mean \pm standard deviation. Data involving two groups following a normal distribution were analyzed using unpaired Student's t-tests, and data with multiple groups were analyzed using one-way ANOVA followed by Tukey's post hoc tests. $\mathrm{P}<0.05$ was considered to indicate a statistically significant difference.

\section{Results}

miRNA expression spectrum. A total of 593 differentially expressed miRNAs were identified and analyzed. The expression levels of a total of nine miRNAs with a fold-change $>2$ and $\mathrm{P}<0.05$ were significantly downregulated, and the expression level of one miRNA was significantly upregulated in the PAH group, compared with the control group (Fig. 1B). The miRNAs 
A

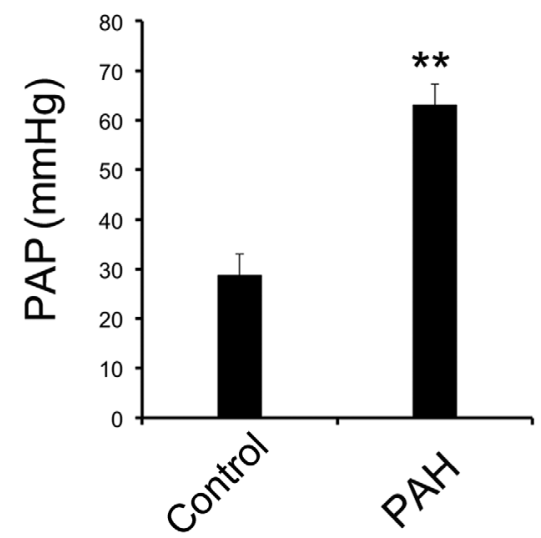

B

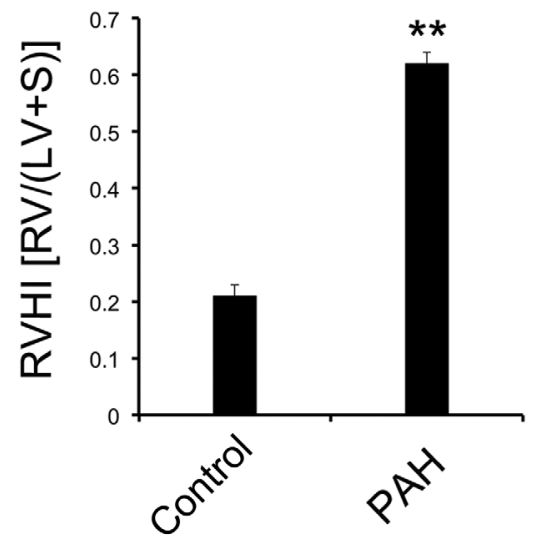

Figure 2. Pulmonary artery pressure and right heart hypertrophy in the PAH animal model. (A) Pulmonary artery pressure in the PAH group was significantly increased compared with the control group. (B) Right heart hypertrophy index in the PAH group was significantly increased compared with the control group. ${ }^{* * *} \mathrm{P}<0.001$ vs. control. $\mathrm{n}=8$ in each group. $\mathrm{PAH}$, pulmonary arterial hypertension; $\mathrm{RV}$, right ventricle; LV, left ventricle; $\mathrm{S}$, ventricular septum.

Table II. Expression levels of the circulating miR-30 family.

\begin{tabular}{llll}
\hline & $\begin{array}{c}\text { Expression in } \\
\text { PAH group } \\
\text { vame }\end{array}$ & & \\
\hline miR-30a-3p & Upregulated & 0.823 & 1.01 \\
miR-30a-5p & Upregulated & 0.963 & 1.11 \\
miR-30b-3p & Upregulated & 0.823 & 1.01 \\
miR-30b-5p & Downregulated & 0.672 & 1.05 \\
miR-30c-1-3p & Upregulated & 0.401 & $2.38^{\mathrm{a}}$ \\
miR-30c-2-3p & Upregulated & 0.406 & 1.89 \\
miR-30c-5p & Downregulated & 0.298 & $2.29^{\mathrm{a}}$ \\
miR-30d-3p & Upregulated & 0.823 & 1.01 \\
miR-30d-5p & Downregulated & $0.046^{\mathrm{b}}$ & $2.66^{\mathrm{a}}$ \\
miR-30e-3p & Downregulated & 0.297 & $2.14^{\mathrm{a}}$ \\
miR-30e-5p & Downregulated & 0.428 & 1.33 \\
\hline
\end{tabular}

The fold-change of miR-30c-1-3p, miR-30c-5p, miR-30d-5p and miR-30e-3p was $>2$; however, only miR-30d-5p exhibited both a fold-change $>2$ and a $\mathrm{P}$-value $<0.05$. ${ }^{\text {aFold-change }}>2$; ${ }^{\text {}} \mathrm{P}<0.05$. $\mathrm{PAH}$, pulmonary arterial hypertension; miR, microRNA.

with downregulated expression levels included miR-30d-5p, miR-151q-3p, miR181a-5p, miR-584-5p, miR-484, miR-4454, miR-3135b, miR-26A-5P and miR-145-5p. The miRNA with an upregulated expression level was miR-1290 (Fig. 1A).

Circulating miR-30 family expression levels. In addition to the aforementioned differentially expressed miRNAs, the expression levels of the circulating miRNA family were also analyzed (Table II). The results revealed the upregulation of miR-30c-1-3p and miR-30c-2-3p, and the downregulation of miR-30c-5p, miR-30d-5p and miR-30e-3p compared with the control group; however, only the altered expression level of miR-30d-5p was significant. Moreover, miR-30a-3p,
miR-30a-5p, miR-30b-3p, miR30b-5p, miR-30d-3p and miR-30e-5p demonstrated a small but not significant fold-change compared with the control group.

Animal model of PAH with increased PAP and RVHI. When the animal model was established, PAP was measured via the jugular vein. RVHI was calculated after the dissection of the heart. Both PAP (Fig. 2A) and RVHI (Fig. 2B) were significantly increased in the PAH group compared with the control group. These results suggested that the PAH model was successfully established.

$R T-q P C R$ results of miR-30 family. As demonstrated in Fig. 1, miR-30d-5p was significantly downregulated in the PAH group compared with the control group; besides, numerous studies have also revealed the association between the miR-30 family and PAH $(15,16)$. Moreover, as a previous study has suggested that the miR-30 family influences vascular smooth muscle cells (19), the expression of the miR-30 family was investigated in the lung tissue of a rat PAH model using RT-qPCR.

The results demonstrated that the expression levels of miR-30a-5p, miR-30b-5p, miR-30c-5p (Fig. 3A), miR-30d-5p, miR-30e-5p, miR-30a-3p (Fig. 3B) and miR-30d-3p (Fig. 3C) were reduced, and the expression levels of miR-30c-2-3p (Fig. 3C) were increased in the PAH group compared with the control group. The expression levels of miR-30c-1-3p (Fig. 3C) and miR-30e-3p (Fig. 3D) were not significantly different between the two groups; although a slight upregulation in miR-30b-3p (Fig. 3C) was observed in the PAH group, compared with the control group, the difference was not significant.

The results of the present study demonstrated that the expression levels of miR-30b-5p were downregulated in both the blood of patients with PAH and the lung tissues of animal models; therefore, miR-30b-5p was selected for analysis in subsequent experiments.

miR-30d-5p inhibits cell toxicity and promotes the apoptosis of PA-SMCs in vitro. RT-qPCR was used to determine 
A

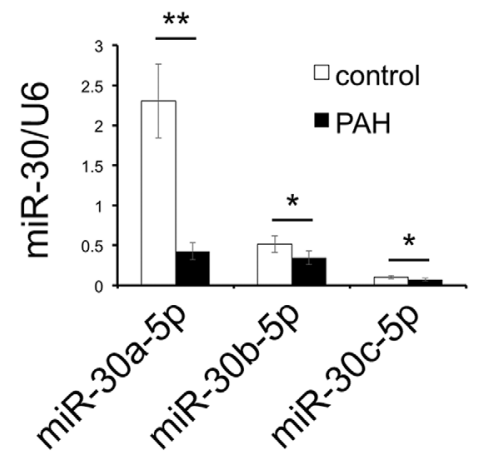

C

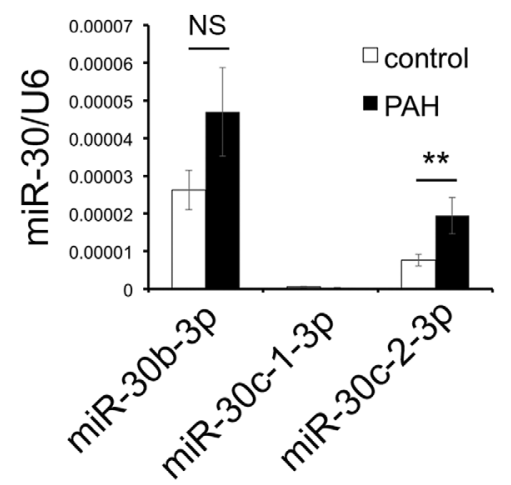

B

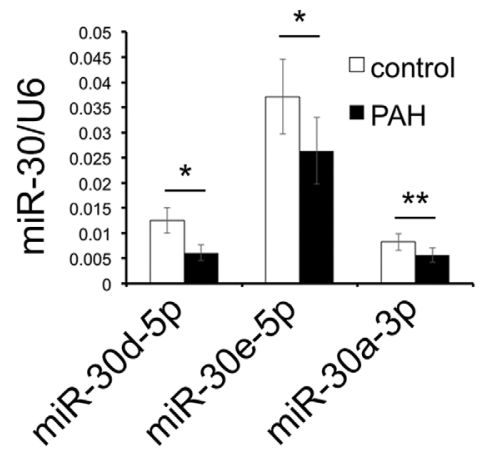

D

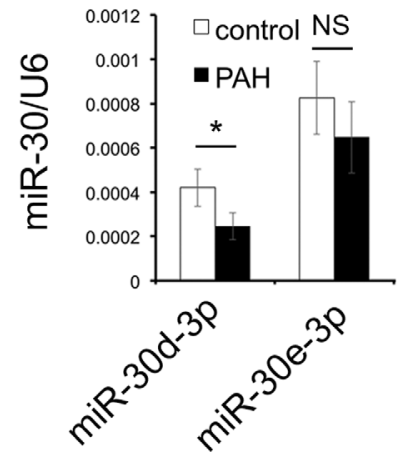

Figure 3. Expression levels of the miR-30 family in a rat PAH model. (A) Downregulation of miR-30a-5p, miR-30b-5p and miR-30c-5p in the PAH group compared with the control group. (B) Downregulation of miR-30d-5p, miR-30e-5p and miR-30a-3p in the PAH group compared with the control group. (C) miR-30c-2-3p expression was significantly upregulated in the PAH group compared with the control group. (D) miR-30d-3p expression was significantly downregulated in the PAH group compared with the control group. $\mathrm{n}=8$ in each group. ${ }^{*} \mathrm{P}<0.05,{ }^{* *} \mathrm{P}<0.01$. miR, microRNA; PAH, pulmonary arterial hypertension; NS, not significant.

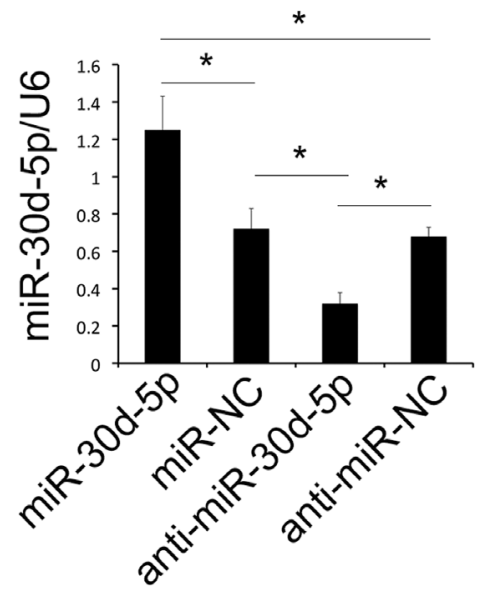

Figure 4. miR-30d-5p expression is increased in the miR-30d-5p mimics group compared with the miR-NC and anti-miR-NC groups. miR-30d-5p expression is decreased in the anti-miR-30d-5p group compared with the miR-NC and anti-miR-NC groups. $n=3$ (experimental repeats) in each group. ${ }^{*} \mathrm{P}<0.05$. miR, microRNA; NC, negative control.

transfection efficiency in PA-SMCs following cell transfection. As demonstrated in Fig. 4, the expression levels of miR-30d-5p were significantly increased in the miR-30d-5p mimics group compared with the miR-NC group. Moreover, the expression levels of miR-30d-5p were significant decreased in the
anti-miR-30d-5p group compared with the anti-miR-NC group (Fig. 4).

An MTT assay was used to assess PDGF-induced PA-SMC toxicity. Following transfection with the miR-30d-5p mimic, the levels of PDGF-induced toxicity of PA-SMCs were significantly decreased, compared with the miR-NC group. Moreover, knockdown of miR-30d-5p resulted in increased levels of toxicity of PA-SMCs, compared with the anti-miR-NC group (Fig. 5A).

Flow cytometry was used to evaluate the levels of cell apoptosis. Notably, following transfection with the miR-30d-5p mimic, the levels of apoptosis of PA-SMCs were significantly increased, compared with the miR-NC group. However, there was no significant difference in the levels of apoptosis of PA-SMCs between the anti-miR-30d-5p and anti-miR-NC groups (Fig. 5C).

miR-30d-5p inhibits the expression of Notch-3. It was previously confirmed that the activation of the Notch-3 pathway participated in the proliferation of PA-SMCs (19). Thus, the expression levels of Notch-3 were determined using western blot analysis. the results of the present study revealed that the expression levels of Notch-3 were significantly decreased in the miR-30d-5p group, compared with all other groups; the expression levels of Notch-3 were significantly increased in the anti-miR-30d-5p group, compared with all other groups (Fig. 5B). 
A

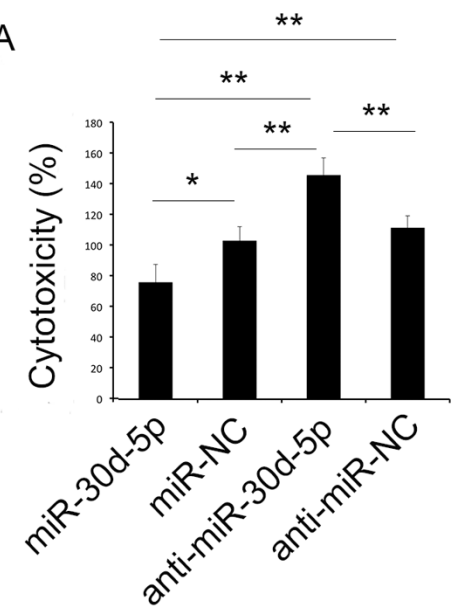

C

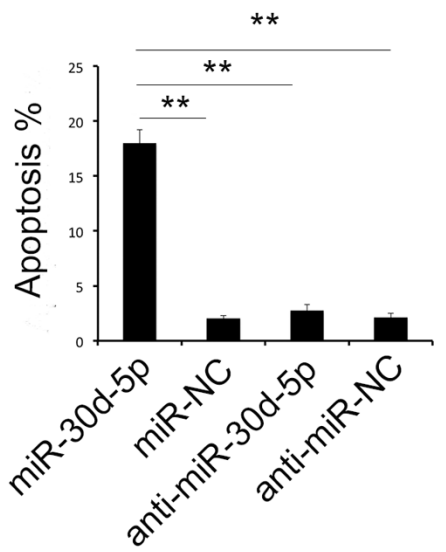

B

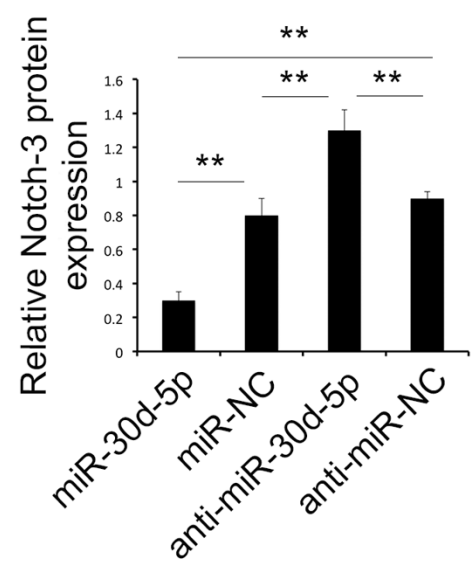

Notch-3

Tubulin

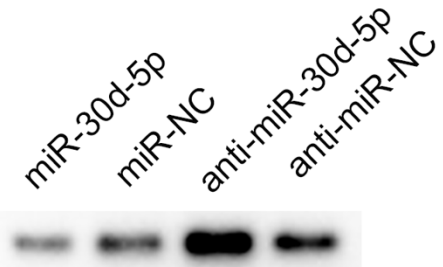

miR-30d-5p

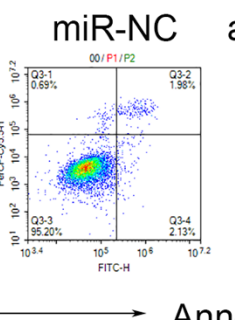

anti-miR-30d-5p anti-miR-NC
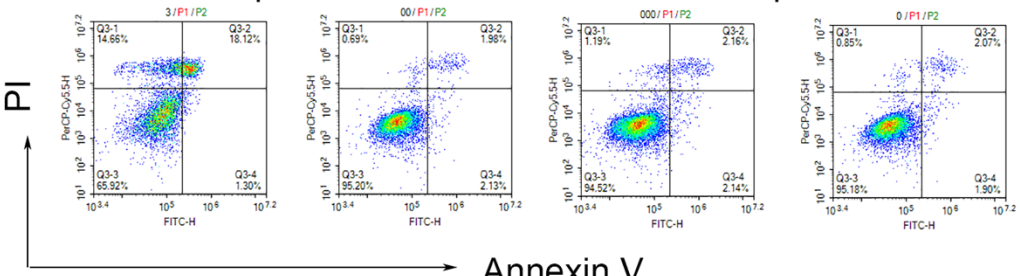

Figure 5. miR-30d-5p inhibits cell toxicity, promotes the apoptosis of PA-SMCs in vitro and inhibits the expression of Notch-3. (A) Cytotoxicity of pulmonary artery vascular smooth muscle cells in the anti-miR-30d-5p group was significantly higher compared with the miR-NC and miR-30d-5p groups. In the miR-30d-5p group, the toxicity was significantly lower compared with the miR-NC and anti-miR-NC groups. (B) Western blot analysis demonstrated that the expression level of Notch-3 was significantly decreased in the miR-30d-5p group compared with the miR-NC and anti-miR-NC group, and increased in the anti-miR-30d-5p group compared with the miR-NC and anti-miR-NC groups. $\mathrm{n}=3$ (experimental repeats) in each group (C) Levels of apoptosis in the miR-30d-5p group were significantly increased compared with all other groups. There was no significant difference between the anti-miR-30d-5p group and miR-NC or anti-miR-NC groups. ${ }^{*} \mathrm{P}<0.05,{ }^{* *} \mathrm{P}<0.01$. miR, microRNA; NC, negative control.

\section{Discussion}

Numerous biological processes involve the participation of miRNAs (20-22). However, the role of various miRNAs in the development of PAH is yet to be fully elucidated. In the present study, miRNAs with differential expression were screened for in patients with PAH using a miRNA microarray. In total, 10 miRNAs with significantly differential expression levels were detected in patients with $\mathrm{PAH}$, compared with the control group.

The results of previous studies have demonstrated changes in the expression levels of miR-151a-3p in a number of diseases $(23,24)$; however, the mechanisms underlying miR-151a-3p in these diseases have not been further studied, to the best of our knowledge. Moreover, it has previously been reported that miR-181a-5p, miR-584-5p, miR-484, miR-145-5p and miR-1290 participated in the modulation of apoptosis and proliferation of numerous cancer types, such as cervical and gastric cancer (25-30). The expression levels of miR-4454 were increased in hypoxic lung alveolar macrophages (31). Furthermore, results of a previous study revealed that miR-181a-5p and miR-4454 participated in cartilage degeneration (32); however, the function of miR-4454 remains to be established. Previous studies have suggested that miR-3135b is associated with heart disease, such as heart failure and acute coronary syndrome $(33,34)$; however, no further study has reported the specific mechanisms underlying miR-3135b. A study on miR-26a-5p expression indicated its association with the metastasis of hepatic cellular cancer (35), but the mechanism remained unclear.

Results of the present study demonstrated that circulating miR-30d-5p expression was significantly reduced in patients with PAH, compared with the control group. Results of previous studies suggested that miR-30d-5p participates in hypoxic-ischemic injury (36), inhibition of prostate cancer cell proliferation (37) and myocardial infarction (38). To the best of our knowledge, this is the first time an association between miR-30d-5p and PAH has been reported.Limitations of current microarray analyses include the generation of false-positive results, and that the levels of miRNAs in circulation may not be identical to those in tissue. Thus, the expression of the miR-30 family, including miR-30d-5p was verified using RT-qPCR analysis in an animal model.

In the animal model, the expression levels of miR-30d-5p were reduced in the lung tissue of the PAH group compared with the control group. In addition, alternate miRNAs in the 
miR-30 family with significant changes in expression levels between the two groups were revealed in the present study. These included reduced expression levels of miR-30a-5p, miR-30b-5p, miR-30c-5p, miR-30e-5p, miR-30a-3p and miR-30d-3p, and an increase in the expression levels of miR-30c-2-3p. In patients with PAH, results of the microarray analysis did not reveal any significant differential expression of the aforementioned miRNAs. These results may be due to the following: i) Species differences between humans and rats; ii) generation of both false negative and positive results using highly efficient microarray technology; and iii) the investigation of circulating miRNAs in patients in the present study, compared with only lung tissues of the animal model. Results of previous studies demonstrated that the expression levels of miR-30a-5p, miR-30b-5p and miR-30a-3p were associated with the proliferation and apoptosis of several types of tumors, such as hepatocellular and renal cell cancer (39-42). Moreover, multiple studies have focused on miR-30c-5p and the associated functions involved in inflammation regulation, tumor migration and invasion $(43,44)$. miR-30e-5p participates in carcinogenesis in different types of tumors through numerous pathways, including the sirtuin 1/JAK/STAT3 signaling and MAPK/nuclear factor of activated T-cells 5 pathway $(45,46)$. Although miR-30d-3p is associated with lung cancer and pancreatic stem cell differentiation, the underlying mechanisms are yet to be elucidated $(47,48)$. Furthermore, miR-30c-2-3p is associated with cell cycle progression in breast cancer and the upregulation of hypoxia-inducible factor- $2 \alpha$ activity in renal cell carcinoma $(49,50)$.

Results of the present study demonstrated that the aforementioned miRNAs exhibited differential expression levels in patients with PAH and animal models; however, the functions of these miRNAs in PAH remain unknown. A number of miRNAs are involved in cell proliferation and apoptosis (51). Results of previous studies have indicated abnormal proliferation and apoptosis in PA-SMCs and PA-ECs in both patients with PAH and animal models $(1,3,6)$; thus, the aforementioned miRNAs may be associated with the proliferation or apoptosis of cells involved in PAH development. Results of the present study revealed that miR-30d-5p overexpression was associated with decreased PA-SMC toxicity and increased apoptosis compared with control groups. These results may provide a theoretical basis for the downregulation of miR-30d-5p in patients with PAH with increased PA-SMC cytotoxicity. In addition, the effects of miR-30d-5p on cell proliferation and apoptosis have been established in numerous other diseases, such as myocardial infarction and lung cancer $(38,52)$.

Several miRNAs have been found to participate in the regulation of PAH. For example, the inhibition of miR-143 inhibited the development of PAH (53). In patients with PAH, miR-124 was downregulated in pulmonary vascular and circulating progenitor endothelial cells (54). In addition, miR-125a-5p ameliorated MCT-induced PAH via tumor growth factor- $\beta 1$ (55). These findings suggest that miRNAs may act as potential therapeutic targets in the treatment of PAH.

Notch signaling participates in multiple physiological vascular processes, such as proliferation and apoptosis (56). Notch-1 is associated with PA-EC proliferation (57), while Notch-3 is highly expressed in PA-SMCs and promotes
PA-SMC proliferation via vascular endothelial growth factor (58). As miR-30d-5p was associated with PA-SMC proliferation and apoptosis, we hypothesized an association between miR-30d-5p and Notch-3. Results of the present study demonstrated that overexpression of miR-30d-5p inhibited Notch-3 expression, while the knockdown of miR-30d-5p induced higher expression levels of Notch-3.

In the present study, the number of patient samples was limited; however, results of the microarray analysis provide novel ideas for further studies. Additional investigations will involve examining potential changes in the markers of proliferation and apoptosis to further verify the observed effects of miR-30d-5p on the regulation of PA-SMC. Furthermore, the sample size of clinical cases will be increased to verify the observed changes of miR-30d-5p in patients with PAH. The mechanisms underlying miR-30d-5p in the regulation of Notch signaling will also be established. Finally, further investigations will also involve the use of miR-30d-5p mimics and the miR-30d-5p inhibitor in animal models of PAH.

In conclusion, miR-30d-5p expression was downregulated in both patients with PAH and animal models. The overexpression of miR-30d-5p resulted in increased cytotoxicity and reduced apoptosis of PA-SMCs. Thus, the mechanisms underlying miR-30d-5p in PAH may be via the Notch-3 signaling pathway.

\section{Acknowledgements}

Not applicable.

\section{Funding}

The present study was supported by grants from the Sichuan Province Science and Technology Support Program, China (grant nos. 2018SZ0180 and 2019YFS0245).

\section{Availability of data and materials}

The datasets generated and/or analyzed during the current study are available in the ArrayExpress database at EMBL-EBI (www.ebi.ac.uk/arrayexpress; accession no. E-MTAB-10932).

\section{Authors' contributions}

FH participated in the analysis and interpretation of the patient data and drafted the manuscript. LQ and HML made substantial contributions to the conception and design of the study. CW performed the examinations and contributed to the acquisition of data. HWL was a major contributor to the experiments. LQ gave final approval of the version to be published and agreed to be accountable for all aspects of the work in ensuring that questions related to the accuracy or integrity of any part of the work are appropriately investigated and resolved. FH and LQ confirm the authenticity of all the raw data. All authors have read and approved the final manuscript.

\section{Ethics approval and consent to participate}

All experiments involving human subjects and animals were approved by the Medical Ethics Committee of West China Second University Hospital of Sichuan University (Chengdu, 
China; approval no. 2015-010). Written informed consent for using the blood samples of patients was obtained from the parents of the patients.

\section{Patient consent for publication}

Parents provided written informed consent for the publication of any data and accompanying images associated with the patients.

\section{Competing interests}

The authors declare that they have no competing interests.

\section{References}

1. Hu F, Liu C, Liu H, Xie L and Yu L: Ataxia-telangiectasia mutated (ATM) protein signaling participates in development of pulmonary arterial hypertension in rats. Med Sci Monit 23. 4391-4400, 2017.

2. Goldberg AB, Mazur W and Kalra DK: Pulmonary hypertension: Diagnosis, imaging techniques, and novel therapies. Cardiovasc Diagn Ther 7: 405-417, 2017.

3. Hansmann G: Pulmonary hypertension in infants, children, and young adults. J Am Coll Cardiol 69: 2551-2569, 2017.

4. McClenaghan C, Woo KV and Nichols CG: Pulmonary hypertension and ATP-sensitive potassium channels. Hypertension 74: 14-22, 2019.

5. Elia D, Caminati A, Zompatori M, Cassandro R, Lonati C, Luisi F, Pelosi G, Provencher S and Harari S: Pulmonary hypertension and chronic lung disease: Where are we headed? Eur Respir Rev 28: 31636088, 2019.

6. Singh I, Oliveira RKF, Naeije R, Rahaghi FN, Oldham WM, Systrom DM and Waxman AB: Pulmonary vascular distensibility and early pulmonary vascular remodeling in pulmonary hypertension. Chest 156: 724-732, 2019.

7. Ye J, Xu M, Tian X, Cai S and Zeng S: Research advances in the detection of miRNA. J Pharm Anal 9: 217-226, 2019.

8. Lin S and Gregory RI: MicroRNA biogenesis pathways in cancer. Nat Rev Cancer 15: 321-333, 2015.

9. Wang S, Cao W, Gao S, Nie X, Zheng X, Xing Y, Chen Y, Bao H and Zhu D: TUG1 Regulates Pulmonary Arterial Smooth Muscle Cell Proliferation in Pulmonary Arterial Hypertension. Can J Cardiol 35: 1534-1545, 2019.

10. Wang Y, Pandey RN, York AJ, Mallela J, Nichols WC, Hu YC, Molkentin JD, Wikenheiser-Brokamp KA and Hegde RS: The EYA3 tyrosine phosphatase activity promotes pulmonary vascular remodeling in pulmonary arterial hypertension. Nat Commun 10: 4143, 2019.

11. Ruffenach G, Umar S, Vaillancourt M, Hong J, Cao N, Sarji S, Moazeni S, Cunningham CM, Ardehali A, Reddy ST, et al: Histological hallmarks and role of Slug/PIP axis in pulmonary hypertension secondary to pulmonary fibrosis. EMBO Mol Med 11: e10061, 2019.

12. Zheng Y, Lu X, Xu L, Chen Z, Li Q and Yuan J: MicroRNA-675 promotes glioma cell proliferation and motility by negatively regulating retinoblastoma 1. Hum Pathol 69: 63-71, 2017.

13. Cheleschi S, Tenti S, Mondanelli N, Corallo C, Barbarino M, Giannotti S, Gallo I, Giordano A and Fioravanti A: MicroRNA-34a and MicroRNA-181a mediate visfatin-induced apoptosis and oxidative stress via NF- $\kappa \mathrm{B}$ pathway in human osteoarthritic chondrocytes. Cells 8: 874, 2019.

14. Wang H, Tan Z, Hu H, Liu H, Wu T, Zheng C, Wang X, Luo Z, Wang J, Liu S, et al: MicroRNA-21 promotes breast cancer proliferation and metastasis by targeting LZTFL1. BMC Cancer 19: $738,2019$.

15. Liu T, Zou XZ, Huang N, Ge XY, Yao MZ, Liu H, Zhang Z and Hu CP: Down-regulation of miR-204 attenuates endothelial-mesenchymal transition by enhancing autophagy in hypoxia-induced pulmonary hypertension. Eur J Pharmacol 863: 172673, 2019.

16. Babicheva A, Ayon RJ, Zhao T, Ek Vitorin JF, Pohl NM, Yamamura A, Yamamura H, Quinton BA, Ba M, Wu L, et al: MicroRNA-mediated downregulation of $\mathrm{K}^{+}$channels in pulmonary arterial hypertension. Am J Physiol Lung Cell Mol Physiol 318: L10-L26, 2020.
17. Livak KJ and Schmittgen TD: Analysis of relative gene expression data using real-time quantitative PCR and the $2(-\Delta \Delta \mathrm{C}(\mathrm{T}))$ Method. Methods 25: 402-408, 2001.

18. Yin Y, Wu X, Yang Z, Zhao J, Wang X, Zhang Q, Yuan M, Xie L, Liu H and He Q: The potential efficacy of R8-modified paclitaxel-loaded liposomes on pulmonary arterial hypertension. Pharm Res 30: 2050-2062, 2013.

19. Liu YF, Spinelli A, Sun LY, Jiang M, Singer DV, Ginnan R, Saddouk FZ, Van Riper D and Singer HA: MicroRNA-30 inhibits neointimal hyperplasia by targeting $\mathrm{Ca}(2+) /$ calmodulin-dependent

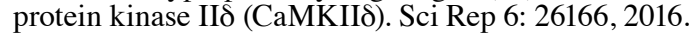

20. Morris HE, Neves KB, Montezano AC, MacLean MR and Touyz RM: Notch3 signalling and vascular remodelling in pulmonary arterial hypertension. Clin Sci (Lond) 133: 2481-2498, 2019.

21. Li L, Zhu X, Shou T, Yang L, Cheng X, Wang J, Deng L and Zheng Y: MicroRNA-28 promotes cell proliferation and invasion in gastric cancer via the PTEN/PI3K/AKT signalling pathway. Mol Med Rep 17: 4003-4010, 2018.

22. Yang Z, Xu J, Zhu R and Liu L: Down-regulation of miRNA-128 contributes to neuropathic pain following spinal cord injury via activation of P38. Med Sci Monit 23: 405-411, 2017.

23. Mundalil Vasu M, Anitha A, Thanseem I, Suzuki K, Yamada K, Takahashi T, Wakuda T, Iwata K, Tsujii M, Sugiyama T and Mori N: Serum microRNA profiles in children with autism. Mol Autism 5: 40, 2014.

24. Zhang K, Wu X, Wang J, Lopez J, Zhou W, Yang L, Wang SE, Raz DJ and Kim JY: Circulating miRNA profile in esophageal adenocarcinoma. Am J Cancer Res 6: 2713-2721, 2016.

25. Yang M, Zhai X, Ge T, Yang C and Lou G: miR-181a-5p Promotes Proliferation and Invasion and Inhibits Apoptosis of Cervical Cancer Cells via Regulating Inositol Polyphosphate-5-Phosphatase A (INPP5A). Oncol Res 26: 703-712, 2018

26. Liu Z, Sun F, Hong Y, Liu Y, Fen M, Yin K, Ge X, Wang F, Chen X and Guan W: MEG2 is regulated by miR-181a-5p and functions as a tumour suppressor gene to suppress the proliferation and migration of gastric cancer cells. Mol Cancer 16: 133, 2017.

27. Li Q, Li Z, Wei S, Wang W, Chen Z, Zhang L, Chen L, Li $\mathrm{B}$, Sun G, Xu J, et al: Overexpression of miR-584-5p inhibits proliferation and induces apoptosis by targeting WW domain-containing E3 ubiquitin protein ligase 1 in gastric cancer. J Exp Clin Cancer Res 36: 59, 2017.

28. Liu J and Li SM: MiR-484 suppressed proliferation, migration, invasion and induced apoptosis of gastric cancer via targeting CCL-18. Int J Exp Pathol 101: 203-214, 2020.

29. Wu J, He Y, Luo Y, Zhang L, Lin H, Liu X, Liu B, Liang C, Zhou Y and Zhou J: miR-145-5p inhibits proliferation and inflammatory responses of RMC through regulating AKT/GSK pathway by targeting CXCL16. J Cell Physiol 233: 3648-3659, 2018.

30. Ma Q, Wang Y, Zhang H and Wang F: miR-1290 contributes to colorectal cancer cell proliferation by targeting INPP4B. Oncol Res 26: 1167-1174, 2018.

31. Armstrong DA, Nymon AB, Ringelberg CS, Lesseur C, Hazlett HF, Howard L, Marsit CJ and Ashare A: Pulmonary microRNA profiling: implications in upper lobe predominant lung disease. Clin Epigenetics 9: 56, 2017.

32. Nakamura A, Rampersaud YR, Sharma A, Lewis SJ, Wu B, Datta P, Sundararajan K, Endisha H, Rossomacha E, Rockel JS, et al: Identification of microRNA-181a-5p and microRNA-4454 as mediators of facet cartilage degeneration. JCI Insight 1: e86820, 2016.

33. Chen F, Yang J, Li Y and Wang H: Circulating microRNAs as novel biomarkers for heart failure. Hellenic J Cardiol 59: 209-214, 2018

34. Wang A, Kwee LC, Grass E, Neely ML, Gregory SG, Fox KAA, Armstrong PW, White HD, Ohman EM, Roe MT, et al: Whole blood sequencing reveals circulating microRNA associations with high-risk traits in non-ST-segment elevation acute coronary syndrome. Atherosclerosis 261: 19-25, 2017.

35. Liang L, Zeng JH, Wang JY, He RQ, Ma J, Chen G, Cai XY and Hu XH: Down-regulation of miR-26a-5p in hepatocellular carcinoma: A qRT-PCR and bioinformatics study. Pathol Res Pract 213: 1494-1509, 2017.

36. Zhao F, Qu Y, Zhu J, Zhang L, Huang L, Liu H, Li S and Mu D: miR-30d-5p plays an important role in autophagy and apoptosis in developing rat brains after hypoxic-ischemic injury. J Neuropathol Exp Neurol 76: 709-719, 2017. 
37. Song Y, Song C and Yang S: Tumor-Suppressive Function of miR-30d-5p in Prostate Cancer Cell Proliferation and Migration by Targeting NT5E. Cancer Biother Radiopharm 33: 203-211, 2018.

38. Jia K, Shi P, Han X, Chen T, Tang H and Wang J: Diagnostic value of miR-30d-5p and miR-125b-5p in acute myocardial infarction. Mol Med Rep 14: 184-194, 2016.

39. Li WF, Dai H, Ou Q, Zuo GQ and Liu CA: Overexpression of microRNA-30a-5p inhibits liver cancer cell proliferation and induces apoptosis by targeting MTDH/PTEN/AKT pathway. Tumour Biol 37: 5885-5895, 2016.

40. Wang C, Cai L, Liu J, Wang G, Li H, Wang X, Xu W, Ren M, Feng L, Liu P, et al: MicroRNA-30a-5p inhibits the growth of renal cell carcinoma by modulating GRP78 expression. Cell Physiol Biochem 43: 2405-2419, 2017.

41. Liu W, Li H, Wang Y,Zhao X, Guo Y, Jin J and Chi R: miR-30b-5p functions as a tumor suppressor in cell proliferation, metastasis and epithelial-to-mesenchymal transition by targeting G-protein subunit $\alpha-13$ in renal cell carcinoma. Gene 626: 275-281, 2017.

42. Qi B, Wang Y, Chen ZJ, Li XN, Qi Y, Yang Y, Cui GH, Guo HZ, $\mathrm{Li} \mathrm{WH}$ and Zhao S: Down-regulation of miR-30a-3p/5p promotes esophageal squamous cell carcinoma cell proliferation by activating the Wnt signaling pathway. World J Gastroenterol 23: 7965-7977, 2017.

43. Ceolotto G, Giannella A, Albiero M, Kuppusamy M, Radu C, Simioni P, Garlaschelli K, Baragetti A, Catapano AL, Iori E, et al: miR-30c-5p regulates macrophage-mediated inflammation and pro-atherosclerosis pathways. Cardiovasc Res 113: 1627-1638, 2017.

44. Cao JM, Li GZ, Han M, Xu HL and Huang KM: miR-30c-5p suppresses migration, invasion and epithelial to mesenchymal transition of gastric cancer via targeting MTA1. Biomed Pharmacother 93: 554-560, 2017.

45. Xu G, Cai J, Wang L, Jiang L, Huang J, Hu R and Ding F: MicroRNA-30e-5p suppresses non-small cell lung cancer tumorigenesis by regulating USP22-mediated Sirt1/JAK/STAT3 signaling. Exp Cell Res 362: 268-278, 2018.

46. Qin X, Li C, Guo T, Chen J, Wang HT, Wang YT, Xiao YS, Li J, Liu P, Liu ZS, et al: Upregulation of DARS2 by HBV promotes hepatocarcinogenesis through the miR-30e-5p/MAPK/NFAT5 pathway. J Exp Clin Cancer Res 36: 148, 2017.

47. Wang K, Chen M and Wu W: Analysis of microRNA (miRNA) expression profiles reveals 11 key biomarkers associated with non-small cell lung cancer. World J Surg Oncol 15: 175, 2017.

48. Coskun E, Ercin M and Gezginci-Oktayoglu S: The role of epigenetic regulation and pluripotency-related microRNAs in differentiation of pancreatic stem cells to beta cells. J Cell Biochem 119: 455-467, 2018.

49. Shukla K, Sharma AK, Ward A, Will R, Hielscher T, Balwierz A, Breunig C, Münstermann E, König R, Keklikoglou I, et al: MicroRNA-30c-2-3p negatively regulates NF- $\kappa$ B signaling and cell cycle progression through downregulation of TRADD and CCNE1 in breast cancer. Mol Oncol 9: 1106-1119, 2015.
50. Mathew LK, Lee SS, Skuli N, Rao S, Keith B, Nathanson KL, Lal $P$ and Simon MC: Restricted expression of miR-30c-2-3p and miR-30a-3p in clear cell renal cell carcinomas enhances HIF $2 \alpha$ activity. Cancer Discov 4: 53-60, 2014.

51. Jacquin S, Rincheval V, Mignotte B, Richard S, Humbert M, Mercier O, Londoño-Vallejo A, Fadel E and Eddahibi S: Inactivation of p53 is sufficient to induce development of pulmonary hypertension in rats. PLoS One 10: e0131940, 2015.

52. Gao L, He RQ, Wu HY, Zhang TT, Liang HW, Ye ZH, Li ZY, Xie TT, Shi Q, Ma J, et al: Expression Signature and Role of miR-30d-5p in Non-Small Cell Lung Cancer: A comprehensive study based on in silico analysis of public databases and in vitro experiments. Cell Physiol Biochem 50: 1964-1987, 2018.

53. Deng L, Blanco FJ, Stevens H, Lu R, Caudrillier A, McBride M, McClure JD, Grant J, Thomas M, Frid M, et al: MicroRNA-143 activation regulates smooth muscle and endothelial cell crosstalk in pulmonary arterial hypertension. Circ Res 117: 870-883, 2015.

54. Caruso P, Dunmore BJ, Schlosser K, Schoors S, Dos Santos C, Perez-Iratxeta C, Lavoie JR, Zhang H, Long L, Flockton AR, et al: Identification of MicroRNA-124 as a major regulator of enhanced endothelial cell glycolysis in pulmonary arterial hypertension via PTBP1 (polypyrimidine tract binding protein) and pyruvate kinase M2. Circulation 136: 2451-2467, 2017.

55. Cai Z, Li J, Zhuang Q, Zhang X, Yuan A, Shen L, Kang K, Qu B, Tang Y, Pu J, et al: miR-125a-5p ameliorates monocrotaline-induced pulmonary arterial hypertension by targeting the TGF- $\beta 1$ and IL-6/STAT3 signaling pathways. Exp Mol Med 50: $1-11,2018$.

56. Thistlethwaite PA, Li $X$ and Zhang $X$ : Notch signaling in pulmonary hypertension. Adv Exp Med Biol 661: 279-298, 2010.

57. Skurikhin EG, Krupin VA, Pershina OV, Pan ES, Ermolaeva LA, Pakhomova AV, Rybalkina OY, Ermakova NN, Khmelevskaya ES, Vaizova OE, et al: Endothelial progenitor cells and Notch-1 signaling as markers of alveolar endothelium regeneration in pulmonary emphysema. Bull Exp Biol Med 166: 201-206, 2018

58. Guo ML, Kook YH, Shannon CE and Buch S: Notch3/VEGF-A axis is involved in TAT-mediated proliferation of pulmonary artery smooth muscle cells: Implications for HIV-associated PAH. Cell Death Discov 4: 22, 2018.

This work is licensed under a Creative Commons Attribution-NonCommercial-NoDerivatives 4.0 International (CC BY-NC-ND 4.0) License. 